www.conferenceie.ase.ro

\title{
WORD EMBEDDINGS IN ROMANIAN FOR THE RETAIL BANKING DOMAIN
}

\author{
Irina RAICU \\ Bucharest University of Economic Studies \\ irina.raicu@ie.ase.ro \\ Nicolas BOITOUT \\ Société Générale European Business Services \\ nicolas.boitout@socgen.com \\ Razvan BOLOGA \\ Bucharest University of Economic Studies \\ razvanbologa@ase.ro \\ Mihai George STURZA \\ Bucharest School of Artificial Intelligence \\ sturzamihai23@gmail.com
}

\begin{abstract}
Word embeddings are a considerable improvement in Natural Language Processing and Deep Learning research fields as a result of their effectiveness for representing textual data in form of fixed-length dense vectors. Currently, domain general pre-trained word embeddings such as Word2Vec and FastText are available in Romanian language. Retail banking domain is a context-sensitive field similar to financial or legal domains where people are using specific terms that are having particular meaning in that specific context. The main goal is to capture retail banking context in order to perform Sentiment Analysis tasks on retail banking reviews in Romanian language. Therefore, this research paper introduces a domainspecific word embeddings called RoBankingFastVec for representing textual data in Romanian language from retail banking field based on online resources such as Wikipedia, retail banking newspapers and books. A research framework is proposed in order to properly conduct the implementation of the retail banking domain-specific word embeddings. The word vectors are obtained by training a FastText model using Skip-gram architecture. By exploring embedding space, the word vectors are evaluated through different tasks related to word embeddings such as Clustering, Similarity, Analogy, Mismatching.
\end{abstract}

Keywords: FastText, Neural Networks, Retail Banking, Vector Representation, Word Embeddings

JEL classification: $\mathrm{C} 6, \mathrm{C} 67$

DOI: $10.24818 / \mathrm{ie} 2020.04 .06$

\section{Introduction}

Word embeddings known as distributed word representations or word vectors have been proposed as an alternative method for representing textual data in feature vectors [1]. Word embeddings can be seen as a Natural Language Processing (NLP) method which is based on a set of techniques of language modeling and feature learning to encode documents, sentences or words from a vocabulary to fixed-length dense vectors of real numbers. In simple terms, word embeddings are a word representation as a vector with dimensions corresponding to the words in the vocabulary and whose values are generated through feature engineering or most recently, unsupervised learning. 
One of the most popular word embeddings models implemented by Google is Word2Vec [2]. Word2Vec was proposed for a better representation of word vectors so that the words similarity is captured more meaningfully by reducing the word vectors dimensionality. The idea behind Word2 $\mathrm{Vec}$ is that instead of considering the words frequency, a classifier is learning how to do a prediction. Hence, Word $2 \mathrm{Vec}$ is a predictive model that learns embeddings through a one hidden layer neural network of variable size by using a predefined number of words before and after a target word (the window size). A large corpus of textual data is given as input to Word2Vec and after learning process, Word2Vec produces a set of word vectors in a low dimensional vector space that are consistent with the context. Recently, Facebook implemented a word embeddings model called FastText [3] as an evolution of Word2Vec for enriching word vectors with sub-word information in the prediction model. Word embeddings such as Word2Vec or FastText could be applied on the own training dataset if there are enough data to be given as input. If the amount of training dataset is limited, available pre-trained word embeddings could be used as transfer learning methods to improve the accuracy of the model. Facebook released FastText pre-trained word embeddings that were trained on Wikipedia for 90 languages [3], including Romanian. In addition, Facebook released a year later another version of FastText pre-trained word embeddings, trained on Common Crawl and Wikipedia [4]. Another pre-trained word embeddings in Romanian can be found at [5] and [6]. These pretrained word embeddings are generic, domain-general being hard to capture domain-specific particularities. In dynamic environments such as financial, legal or retail banking, people are using domain-related terms having a specific meaning in that context. The context plays an important role in sentiment analysis tasks in order to classify the sentiment in an accurate way. Therefore, several studies proposed domain-specific word embeddings. For instance, a domain-specific word embeddings in financial field that outperformed the domain-general Word2Vec embeddings has been proposed in [7]. In this paper, RoBankingFastVec based on FastText model, word embeddings for the retail banking domain in Romanian language, are proposed and evaluated. Textual data is collected from several online resources specific to retail banking domain in Romanian language yielding a corpus of $\mathbf{3 2 3 . 0 9 7}$ of Romanian words. The quality of the proposed word embeddings is evaluated using different tasks related to word embeddings such as Clustering, Similarity, Analogy, and Mismatching.

\section{Research Framework for development of RoBankingFastVec}

A research framework composed by four stages is proposed to develop RoBankingFastVec, word embeddings in Romanian language specific to retail banking context in order to be further used in sentiment analysis tasks of reviews in Romanian language. The research framework is composed by the following stages: Data Sources Identification, Data Sources Aggregation, Training Process and Exploration of the embedding space. These stages are described in the following sub-sections.

\subsection{Data Sources Identification}

In this stage, several online resources that contain retail banking related text were identified including websites in Romanian language from banks and financial institutions, newspapers, blogs and Wikipedia pages with specific to retail banking context. In addition, a financial banking education book which contains a relevant number of retail banking related words was considered as identified data source. Interviews, redactors or analysts opinions were not considered for extraction. Online resources considered for extraction are presented in Table 1.

Table 1. Identified data sources 
www.conferenceie.ase.ro

\begin{tabular}{|l|l|l|l|l|l|l|l|l|}
\hline $\begin{array}{l}\text { Data } \\
\text { Source }\end{array}$ & $\begin{array}{l}\text { Wikipedia } \\
\text { Domain: } \\
\text { Retail } \\
\text { Banking } \\
\text { Romanian } \\
\text { language }\end{array}$ & $\begin{array}{l}\text { Banstitutions } \\
\text { Financial } \\
\text { instites } \\
\text { and } \\
\text { BNR }\end{array}$ & Finzoom & Conso & $\begin{array}{l}\text { Vreau } \\
\text { credit }\end{array}$ & BugetulFamiliei & Efin & $\begin{array}{l}\text { Manualul } \\
\text { Practic } \\
\text { pentru } \\
\text { utilizatorul } \\
\text { de servicii } \\
\text { financiare }\end{array}$ \\
\hline $\begin{array}{l}\text { Number } \\
\text { of } \\
\text { words }\end{array}$ & 113760 & 82917 & 20323 & 24877 & 8451 & 8901 & 7564 & 56.304 \\
\hline
\end{tabular}

\subsection{Data Sources Aggregation}

After data sources identification, the next stage is related to data sources aggregation. The first step is to extract the relevant retail banking text in Romanian language from identified data sources. Using Scrapy as framework for data extraction, several web scrapers were created for performing the data extraction yielding a corpus of 323.097 Romanian words related to retail banking context stored in a CSV file.

\subsection{Training Process}

This stage is composed by two steps: corpus preprocessing and the model configuration.

\subsubsection{Corpus Preprocessing}

In this step, several tasks for cleaning the text from the corpus are performed. In most cases, in Romanian online resources, diacritics are omitted. Several websites don't use diacritics in their text, even most popular websites such as the Citi bank website ${ }^{1}$. Hence, the first step in preprocessing the text from the corpus is to remove Romanian special characters which are refereed as diacritics. Then numbers, symbols, spaces and non-ASCII characters were eliminated. In addition, a lemmatization function was implemented in order to preserve the relative meaning of words when building the word embeddings.

\subsubsection{Model Configuration}

Obtaining quality-based word embeddings mainly depends on configuration of model parameters. For training RoBankingFastVec, the FastText model proposed by [3] is chosen following the implementation supported by Python library, Gensim [8]. FastText model is based on a neural network of one hidden layer that takes as input the data corpus and provides as output produces a set of word vectors of real numbers. To capture the context and to create meaningful semantic relationships between words, FastText model requires several parameters as input that are essential as directly influence the output. The FastText parameters configuration are detailed in Table 2. The architecture model chosen to train the FastText model for obtaining RoBankingFastVec word embeddings was Skip-gram that works better for smaller corpuses [9]. RoBankingFastVec receives as input the given corpus and learns embeddings by starting with an initial set of embeddings with random then shifting and take the embeddings of words that occurs nearby in texts according to the model configuration parameters. The dimension of the context window influences directly the semantic relationships of words. Using shorter context windows will generate similar words whilst larger context windows will generate topically related words. A good example of this aspect can be found in [13].

\footnotetext{
${ }^{1}$ http://www.citibank.ro/romania/
} 
www.conferenceie.ase.ro

Table 2. FastText Configuration Model Parameters

\begin{tabular}{|c|c|c|}
\hline $\begin{array}{l}\text { FastText } \\
\text { Parameter } \\
\text { Gensim }\end{array}$ & Description & Value \\
\hline sentences & Corpus in form of tokenized text. & $\begin{array}{l}\text { Corpus composed by } \\
\text { 323.097 Romanian words }\end{array}$ \\
\hline size & $\begin{array}{l}\text { Size of the hidden layer which determines the } \\
\text { dimension of the dense output vector. }\end{array}$ & 100 \\
\hline window & $\begin{array}{l}\text { Context window used for collecting words } \\
\text { counts. }\end{array}$ & 10 \\
\hline sg & $\begin{array}{l}\text { Option for choosing the architecture model } \\
\text { between CBOW and Skip-gram. The default } \\
\text { value is set to } 0 \text { for training a CBOW, } 1 \text { is for } \\
\text { Skip-gram. }\end{array}$ & 1 \\
\hline min_count & $\begin{array}{l}\text { rare words from the corpus that are ignored for } \\
\text { the training process. }\end{array}$ & 5 \\
\hline sample & Down sampling of words & $1 \mathrm{e}-2$ \\
\hline iter & $\begin{array}{l}\text { Number of epochs over the samples used to } \\
\text { train the model }\end{array}$ & 100 \\
\hline
\end{tabular}

\subsection{Exploration of the embedding space}

For performing Natural Language Processing and Sentiment Analysis tasks, the quality of the word embeddings is essential. The previous stages were dedicated to build RoBankingFastVec and this stage evaluates the quality of the generated word embeddings in Romanian language for retail banking domain. There is not a quantitative baseline criterion for evaluating the quality of word embeddings. Even so, in literature, various intrinsic or extrinsic methods have been proposed for word embeddings quality evaluation such as: POS tagging, analogy, similarity and sentiment classification [10]. In this paper, for assessing the quality of the semantic relationships between retail banking-specific concepts different tasks related to word embeddings such as Clustering, Similarity, Analogy, and Mismatching, are used.

\subsubsection{Clustering}

Word with similar meaning are grouped nearby in the vector space. Visualization of embedding space allows to verify if the words with similar meaning in the context of retail banking are grouped nearby each other by forming clusters. The embeddings space can be visualized by using T-SNE (acronym of t-distributed stochastic neighbor embedding visualization algorithm) [11]. For exemplification, considering two words 'dobanda' (Romanian translation of English word 'interest') and 'comision' (translation of English word 'comission'), the similar Romanian words (top 5 words) in meaning from words 'dobanda', and 'comision' are grouped and the similar words in meaning are also close together in the RoBankingFastVec word embeddings space.

\subsubsection{Similarity}

Cosine similarity metric or dot product is a widely used metric for exploring the similarity of words from the embeddings space. To determine the cosine similarity, a query is performed to the trained model in order to return the 5 words closest. For return the top 5 words similar in meaning to "dobanda" (from English "interest rate"), the following query was executed:

$$
f t \_ \text {model.wv.most_similar(positive }=[\text { 'dobanda']) }
$$

\footnotetext{
${ }^{2}$ https://radimrehurek.com/gensim/models/fasttext.html
} 
The results of this query are presented in Table 3.

Table 3. Top 5 words closet to 'dobanda' (cosine distance)

\begin{tabular}{|l|l|}
\hline Words closest to "dobanda" & Cosine distance \\
\hline fixa (Translation from English "fix") & .73 \\
\hline rata (Translation from English "credit rate") & .72 \\
\hline lunar (Translation from English "monthly") & .66 \\
\hline variabila (Translation from English "variable") & .66 \\
\hline credit (Translation from English "loan") & .60 \\
\hline
\end{tabular}

As illustrated, RoBankingFastVec model even if it is composed by only raw textual data and it was trained in an unsupervised way, RoBankingFastVec managed to learn different semantic relationship between words in retail banking context. Also, for the out-of-vocabulary words, the model can adapt and make correct semantic correlations.

\subsubsection{Analogy}

In natural language processing, word pairs are often used. Word analogies are word pairs with semantic meaning [12] which can be determined using vector offset method [2]. For instance, the vector operation of words: Science + Art - Tesla $=$ Michelangelo. This means that the Michelangelo is determined by measuring how closer the vector is to the operation of word pairs. Considering two words pairs $\mathrm{P}_{1}$ composed by vectors $\mathrm{w}_{1}$ and $\mathrm{w}_{2}$ and pair $\mathrm{P}_{2}$ composed by vectors $\mathrm{v}_{1}$ and $\mathrm{v}_{2}$, table 4 illustrates how word analogies are organized within embedding space by RoBankingFastVec.

Table 4. Word analogies for RoBankingFastText

\begin{tabular}{|l|l|l|l|}
\hline \multicolumn{1}{|c|}{$\overrightarrow{\mathrm{w} 1}$} & \multicolumn{1}{|c|}{$\overrightarrow{\mathrm{w} 2}$} & $\overrightarrow{\mathrm{v} 1}$ & \multicolumn{1}{c|}{$\overrightarrow{\mathrm{v} 2}$} \\
\hline dobanda (interest) & credit (loan) & comision(commission) & card (card) \\
\hline polita (policy) & viata (life) & credit (loan) & ipotecar (real estate) \\
\hline card (card) & posesor (owner) & asigurare (insurance) & asigurat (insurant) \\
\hline
\end{tabular}

\subsubsection{Mismatching}

The aim of the mismatching task is to test the model if it's able to discriminate between different retail banking concepts. In other words, the model should be able to identify from a list of words the word which is not related to that list. To perform this type of query, the model is calculating in the embedding space the cosine similarity for each word in the list and all other words and returns an average result. The word which has the lowest cosine distance value is considered as a no-matching word. Table 5 shows the responses of the RoBankingFastVec model to the mismatching queries.

Table 5. Mismatching queries for RoBankingFastVec model

\begin{tabular}{|l|l|}
\hline \multicolumn{1}{|c|}{ List of words } & \multicolumn{1}{c|}{ Response } \\
\hline moneda, dobanda, rata, fixa, variabila & moneda \\
\hline asigurare, prima, polita, viata, bacnota & bacnota \\
\hline comision, taxa, cost, fngcimm, perceput & fngcimm \\
\hline
\end{tabular}


www.conferenceie.ase.ro

\section{Conclusions}

Word embeddings are a powerful representation of textual data widely used in Natural Language Processing and Deep Learning research fields. This research paper presented RoBankingFastVec, a domain-specific word embeddings for retail banking field from online sources in Romanian language. These custom word embeddings are available on GitHub ${ }^{3}$. The research framework proposed for generating RoBankingFastVec is presented. Textual data specific to retail banking context is extracted from several online resources yielding a corpus of 323.097 of Romanian retail-banking specific words. RoBankingFastVec is trained following FastText implementation based on skip-gram architecture. The quality of the trained embeddings was evaluated through different tasks and the results showed that the RoBankingFastVec encode meaningful semantically relationships in retail banking domain.

So far, in the current literature, there have not been proposed word embeddings for retailbanking in Romanian language. In the future, RoBankingFastVec will be used as a transfer learning method in sentiment analysis tasks of retail banking reviews in Romanian language.

\section{References}

[1]. Bengio, Y. \& Ducharme, Réjean \& Vincent, Pascal \& Jauvin, Christian. (2006). Neural Probabilistic Language Models.

[2]. Mikolov, Tomas \& Corrado, G.s \& Chen, Kai \& Dean, Jeffrey. (2013). Efficient Estimation of Word Representations in Vector Space. 1-12.

[3]. Bojanowski Piotr, Grave Edouard, Joulin Armand, Mikolov Tomas. (2016). Enriching word vectors with subword information. arXiv preprint arXiv:1607.04606

[4]. Grave Edouard, Bojanowski Piotr, Gupta Prakhar, Joulin Armand, Mikolov Tomas, (2018). Learning Word Vectors for 157 Languages. LREC

[5] Pais, V. \& Tufis, Dan. (2018). Computing distributed representations of words using the corola corpus. Proceedings of the Romanian Academy Series A - Mathematics Physics Technical Sciences Information Science. 19. 185-191.

[6] Devlin, Jacob \& Chang, Ming-Wei \& Lee, Kenton \& Toutanova, Kristina. (2018). BERT: Pretraining of Deep Bidirectional Transformers for Language Understanding

[7]. Li Q., Shah S., (2017). Learning Stock Market Sentiment Lexicon and Sentiment-oriented Word Vector from StockTwits, Proceedings of the 21st Conference on Computational Natural Language Learning (CoNLL 2017), Association for Computational Linguistics, Vancouver, BC, Canada pp. $301-310$

[8]. Řehůřrek, Sojka, (2010). Software framework for topic modelling with large corpora. In: Proceedings of the LREC 2010 Workshop on New Challenges for NLP Frameworks, pp. 45-50. ELRA, Valletta, Malta

[9]. Enriquez, Fernando \& Troyano, José \& López-Solaz, Tomás. (2016). An approach to the use of word embeddings in an opinion classification task. Expert Systems with Applications.

[10]. Schnabel T., I. Labutov, D. Mimno, T. Joachims, (2015). Evaluation methods for unsupervised word embeddings, Proceedings of the Conference on Empirical Methods in Natural Language Processing (EMNLP), Association for Computational Linguistics, Lisbon, Portugal, pp. 298-307

[11]. Van Der Maaten, L.J.P., Hinton, G.E., (2008). Visualizing high-dimensional data using T-SNE, 2008.

[12]. Chen, Juntian \& Tao, Yubo \& Lin, Hai. (2018). Visual Exploration and Comparison of Word Embeddings. Journal of Visual Languages \& Computing. 48.

[13]. Levy, O. and Goldberg, Y. (2014a). Dependency-based word embeddings. In ACL 2014

\footnotetext{
${ }^{3}$ https://github.com/irina-raicu/custom-word-embeddings
} 